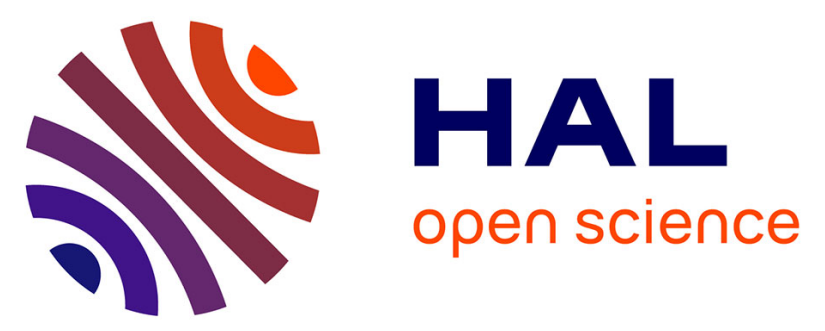

\title{
Exposés et Tables Rondes du Colloque sur les Supraconducteurs Aussois, 10, 11 et 12 mars 1969 organisé par Le Comité d'Action Concertée Métallurgie de la DGRST et l'aide financière de la Division Métaux et Alliages de la DRME
}

Yves A. Rocher, Étienne Guyon

\section{To cite this version:}

Yves A. Rocher, Étienne Guyon. Exposés et Tables Rondes du Colloque sur les Supraconducteurs Aussois, 10, 11 et 12 mars 1969 organisé par Le Comité d'Action Concertée Métallurgie de la DGRST et l'aide financière de la Division Métaux et Alliages de la DRME. Revue de Physique Appliquée, 1969, 4 (4), pp.543-544. 10.1051/rphysap:0196900404054300 . jpa-00243327

HAL Id: jpa-00243327 https://hal.science/jpa-00243327

Submitted on 1 Jan 1969

HAL is a multi-disciplinary open access archive for the deposit and dissemination of scientific research documents, whether they are published or not. The documents may come from teaching and research institutions in France or abroad, or from public or private research centers.
L'archive ouverte pluridisciplinaire HAL, est destinée au dépôt et à la diffusion de documents scientifiques de niveau recherche, publiés ou non, émanant des établissements d'enseignement et de recherche français ou étrangers, des laboratoires publics ou privés. 
Exposés et Tables Rondes

$\mathrm{du}$

\title{
COLLOQUE SUR LES MATÉRIAUX SUPRACONDUCTEURS
}

\author{
Aussois, ro, I I et I2 mars I969 \\ organisé par \\ Le Comité d'Action Concertée Métallurgie de la DGRST \\ et 1'aide financière de la Division Métaux et Alliages de la DRME
}

\author{
AVANT-PROPOS
}

La supraconductivité paraît être en mesure d'apporter une solution techniquement possible et économiquement rentable dans de très nombreux domaines où les besoins actuels et futurs nécessitent soit la mise en œuvre des propriétés uniques des supraconducteurs, soit la recherche de progrès importants dans les performances, le coût ou les conditions d'exploitation d'appareillages très divers.

En présence de ces possibilités variées et prometteuses, il a semblé utile aux organisateurs du colloque de réunir les représentants des laboratoires industriels, publics, semi-publics et universitaires intéressés par l'étude, la fabrication et la mise en œuvre des supraconducteurs, et de faire le point sur l'aspect plus particulier des matériaux supraconducteurs.

Le nombre de cent trente-six participants originaires d'une trentaine de laboratoires est significatif de l'intérêt porté à la supraconductivité. Le caractère très large de cette représentation a permis d'aborder l'ensemble des problèmes avec une connaissance fondamentale des phénomènes mis en jeu aussi bonne que possible et une expérience couvrant tous les domaines de la fabrication et des applications des matériaux supraconducteurs.

Le programme comportait à la fois des exposés et des tables rondes. Les exposés, de caractère général et didactique, avaient été choisis parmi les thèmes les plus actuels, fondamentaux ou appliqués. Les tables rondes, dont les thèmes avaient été retenus à la suite d'une consultation préalable, se rapportaient essentiellement aux différents domaines dans lesquels les chercheurs présents se trouvaient largement engagés. On trouvera, à côté des sujets touchant aux supraconducteurs, une table ronde sur l'équipement cryogénique dont il est inutile de souligner le caractère essentiel et quasiment prioritaire si l'on pense aux développements de la supraconductivité. Conduites non pas comme une succession d'exposés mais comme une discussion sur les points particuliers les plus importants, ces tables rondes devaient permettre tout à la fois le recensement des équipes, méthodes, appareillages et idées, des prises de contact entre chercheurs et le choix des orientations de recherche à développer et d'actions concertées.

L'enthousiasme et la bonne volonté des participants et conférenciers ont garanti à cette rencontre le succès que l'inexpérience et l'immodestie des ambitions des organisateurs rendaient difficile à espérer. Pour ne citer qu'un exemple, la table ronde " Piégeage des lignes de vortex » avait fait apparaître une approche du problème certes liée à des idées brillantes mais dispersée et inefficace. Une nouvelle réunion a permis depuis lors d'aboutir à un programme entièrement concerté entre une dizaine de laboratoires sur des objectifs réalistes et plus prometteurs. Il faut espérer que dans d'autres domaines le même chemin sera suivi. 
On trouvera dans les pages qui suivent le texte des exposés et les comptes rendus des tables rondes. Certaines idées y figurent, sans toute la rigueur scientifique habituellement attachée aux publications de ce journal. Ceci est bien sûr lié à la nature particulière de la réunion d'Aussois, et ces propos doivent être accueillis avec la réserve qui s'impose, encore qu'ils manifestent un indéniable courant d'opinion.

Nous remercions la Direction des Euvres Sociales du C.N.R.S. et tout particulièrement M. Fourdan, directeur du Centre Paul-Langevin, et le personnel de ce Centre, pour la qualité de leur accueil.

Le Comité d'Organisation :

Yves A. Rocher et Étienne Guyon. 